\title{
Avaliação da usabilidade de um aplicativo móvel para o autocuidado de pacientes com diabetes
}

\author{
Assessment of the usability of a mobile application for self-care for patients with diabetes \\ Evaluación de la usabilidad de una aplicación móvil para el autocuidado de pacientes con diabetes
}

Recebido: 21/01/2022 | Revisado: 29/01/2022 | Aceito: 05/02/2022 | Publicado: 07/02/2022

\author{
Débora Almeida Silveira Sobral \\ ORCID: https://orcid.org/0000-0001-9620-1111 \\ Universidade Federal de Sergipe, Brasil \\ E-mail: enfdeborasobral@gmail.com \\ Adicinéia Aparecida de Oliveira \\ ORCID: https://orcid.org/0000-0002-1551-1992 \\ Universidade Federal de Sergipe, Brasil \\ E-mail: adicineia@dcomp.ufs.br \\ Joseilze Santos de Andrade \\ ORCID: https://orcid.org/0000-0003-0488-2840 \\ Universidade Federal de Sergipe, Brasil \\ E-mail: joseilzesa@gmail.com \\ Jonathan Kelvin de Jesus Santos \\ ORCID: https://orcid.org/0000-0001-5500-4277 \\ Universidade Federal de Sergipe, Brasil \\ E-mail: jnthn.klvn.s@gmail.com
}

\begin{abstract}
Resumo
Este estudo objetivou avaliar a usabilidade de um aplicativo móvel para o autocuidado de pacientes com diabetes. Tratase de uma pesquisa metodológica, realizada em três ambulatórios públicos referências em diabetes no Estado de Sergipe. A coleta de dados ocorreu entre julho e setembro de 2021. Foram incluídos 77 pacientes com diabetes, com idade entre 18 e 83 anos. Destes, 2,6\% afirmaram apresentar deficiência visual total. Após conhecerem e testarem o aplicativo DiaVision, os participantes responderam um formulário sobre a avaliação da usabilidade. Foram descritas as frequências absolutas e relativas das variáveis categóricas e a moda e intervalo interquartil das variáveis contínuas. Utilizou-se os Testes de Mann-Whitney e Kruskal-Wallis para avaliar a hipótese de igualdade de moda. Entre os entrevistados, 90,1\% afirmaram que gostariam de ter o aplicativo; $62,3 \%$ avaliaram a satisfação máxima com o aplicativo e 53,2\% responderam que usariam o aplicativo sempre. A principal sugestão de melhoria foi quanto à acessibilidade $(35,1 \%)$, apesar de 45,5\% afirmarem não haver necessidade de melhorias. A maioria declarou que o DiaVision contribuiu com o conhecimento sobre o diabetes e a melhor funcionalidade foi o registro dos valores de glicemia. A idade e o grau de deficiência visual interferiram na facilidade no uso do aplicativo, como também no índice de recomendação do aplicativo. Conclui-se que o aplicativo demonstrou um elevado índice de aprovação, inclusive com altas taxas de frequência de uso diário e de índices de recomendação por parte dos usuários.
\end{abstract}

Palavras-chave: Diabetes Mellitus; Inclusão digital; Autocuidado.

\begin{abstract}
This study aimed to evaluate the usability of a mobile application for self-care for patients with diabetes. This is a methodological research, carried out in three public diabetes clinics in the state of Sergipe. Data collection took place between July and September 2021. 77 patients with diabetes, aged between 18 and 83 years, were included. Of these, $2.6 \%$ said they had total visual impairment. After learning about and testing the DiaVision application, the participants answered a form on the usability assessment. Absolute and relative frequencies of categorical variables and mode and interquartile range of continuous variables were described. The Mann-Whitney and Kruskal-Wallis tests were used to assess the hypothesis of equality of mode. Among respondents, $90.1 \%$ said they would like to have the app; $62.3 \%$ rated maximum satisfaction with the app and 53.2\% responded that they would use the app always. The main suggestion for improvement was regarding accessibility (35.1\%), although $45.5 \%$ stated that there was no need for improvement. The majority stated that DiaVision contributed with their knowledge about diabetes and the best functionality was the recording of blood glucose values. Age and degree of visual impairment interfered in the ease of use of the app, as well as in the app's recommendation rate. It is concluded that the application showed a high approval rate, including high rates of frequency of daily use and recommendation rates by users.
\end{abstract}

Keywords: Diabetes Mellitus; Digital inclusion; Self-care. 


\begin{abstract}
Resumen
Este estudio tuvo como objetivo evaluar la usabilidad de una aplicación móvil para el autocuidado de pacientes con diabetes. Se trata de una investigación metodológica, realizada en tres clínicas públicas de diabetes del estado de Sergipe. La recogida de datos se realizó entre julio y septiembre de 2021. Se incluyeron 77 pacientes con diabetes, con edades comprendidas entre 18 y 83 años. De estos, el $2.6 \%$ dijo que tenía una discapacidad visual total. Después de conocer y probar la aplicación DiaVision, los participantes respondieron un formulario sobre la evaluación de usabilidad. Se describieron las frecuencias absolutas y relativas de las variables categóricas y la moda y rango intercuartílico de las variables continuas. Se utilizaron las pruebas de Mann-Whitney y Kruskal-Wallis para evaluar la hipótesis de igualdad de moda. Entre los encuestados, el 90,1\% dijo que le gustaría tener la aplicación; El 62,3\% calificó la máxima satisfacción con la aplicación y el 53,2\% respondió que usaría la aplicación siempre. La principal sugerencia de mejora fue la accesibilidad (35,1\%), aunque el 45,5\% afirmó que no era necesario mejorar. La mayoría afirmó que DiaVision contribuyó con su conocimiento sobre la diabetes y la mejor funcionalidad fue el registro de los valores de glucosa en sangre. La edad y el grado de discapacidad visual interfirieron en la facilidad de uso de la aplicación, así como en la tasa de recomendación de la aplicación. Se concluye que la aplicación mostró una alta tasa de aprobación, incluyendo altas tasas de frecuencia de uso diario y tasas de recomendación por parte de los usuarios.
\end{abstract}

Palabras clave: Diabetes Mellitus; Inclusión digital; Autocuidado.

\title{
1. Introdução
}

O Diabetes Mellitus (DM) é uma doença crônica e complexa, provocada pela deficiência da produção ou da ação da insulina. Na falta de controle dos níveis glicêmicos, o DM pode gerar diversas complicações (International Diabetes Federation, 2019). Uma das principais complicações é a chamada retinopatia diabética, consequência da hiperglicemia crônica que causa danos aos capilares da retina provocando obstruções ou vazamentos e ocorre em cerca de $75 \%$ dos pacientes após 15 anos de desenvolvimento do DM (International Diabetes Federation, 2019; Bourne et al., 2021a).

Dentro desse contexto, o DM demanda cuidados continuados, estratégias de prevenção e redução de risco, principalmente, o autocuidado. Porém, o autocuidado é praticado apenas por uma minoria da população. Dados globais demonstram que, em 2020, 1,1 bilhão de pessoas vivem com perda de visão e, destes, 90\% são evitáveis ou tratáveis. Além disto, medidas de autocuidado poderiam reduzir $35 \%$ os casos de amputação provocada pela neuropatia diabética (International Diabetes Federation, 2019; Bourne et al., 2021b).

O autocuidado também está associado a melhora no controle glicêmico, percebida com a redução significativa da hemoglobina glicada (HbA1C). Um estudo comparou a efetividade de intervenções de autocuidado em dois grupos de pacientes. Os autores observaram que houve uma redução da $\mathrm{HbA} 1 \mathrm{C}$ de $0,74 \%$ nos pacientes que participaram das intervenções e de $0,17 \%$ nos pacientes que não participaram (Chrvala et al., 2016).

Outro benefício das intervenções do autocuidado no controle da glicemia, como dieta hipoglicêmica e/ou uso correto das medicações, é a possibilidade de reduzir o início da retinopatia diabética em $76 \%$ e sua progressão em $54 \%$ dos casos (Bourne et al., 2021a). Ademais, reduz a menos de 5\% o risco de cegueira pela retinopatia diabética quando o diagnóstico é realizado em tempo adequado e o tratamento feito de modo correto, antes que alterações irreversíveis possam se instalar (Sociedade Brasileira de Diabetes, 2015).

Em relação à deficiência visual devido ao diabetes, Sabanayagam et al. (2019) publicaram uma revisão sistemática com estudos entre 1980 e 2018, cuja incidência anual de retinopatia diabética variou entre 2,2 e 12,7\% na população incluída nos estudos. Ainda demonstraram uma taxa anual de piora da acuidade visual que variou de 3,4 a 12,3\%, com os piores resultados encontrados em indivíduos com doença de gravidade moderada, comparados àqueles sem doença. Trata-se, portanto, de uma condição com grande impacto no custo total para os sistemas de saúde, dada a alta prevalência do DM associada à elevadas taxas de progressão anual.

Uma das principais formas de ensinar o autocuidado aos pacientes com diabetes é por meio da utilização de inovações tecnológicas, visto que essas ferramentas têm, rapidamente, se incorporado à rotina diária das pessoas. Desde as atividades mais simples até as mais complexas, em todos os extratos socioeconômicos, há uma tendência da população, em geral, à adesão de 
diversas ferramentas tecnológicas para a realização de tarefas, antes realizadas de forma menos prática, o que gerava mais gastos desnecessários de tempo e recursos (Kao \& Liebovitz, 2017).

Essa realidade não pode ser diferente em relação à saúde, área fundamental da existência humana e que, naturalmente, atrai inovações que podem contribuir com uma melhor qualidade de vida da população. Ao longo das últimas décadas, observouse diversas ações no sentido de inserir as inovações tecnológicas ao cuidado voltadas aos portadores de qualquer condição que afeta a saúde (Sim, 2019).

A tecnologia em saúde tem potencializado vários desfechos, a exemplo de melhorias no processo de comunicação entre profissionais de saúde, tanto no âmbito do ensino quanto da assistência. Tem ainda aprimorado o acesso à informação em saúde, mesmo que, em alguns cenários, a qualidade da informação seja questionável (Kao \& Liebovitz, 2017). A utilização de softwares para dispositivos móveis em saúde é uma tendência. Estudos têm demonstrado que em diversas doenças crônicas, a utilização deste tipo de tecnologia, em comparação ao cuidado padrão, demonstra maior eficácia nos desfechos finais, incluindo maiores índices de sucesso no autocuidado (Birkhoff \& Smeltzer, 2017).

Dentre as pessoas com deficiência visual, uma etiologia adquire importância pela alta prevalência na sociedade: pessoas com diabetes com algum grau de redução de acuidade visual provocada, principalmente, pela retinopatia diabética (Lechner, O’leary \& Stitt, 2017). Diante do elevado número de pessoas com deficiência visual, a reflexão sobre acessibilidade é um ponto urgente e de relevância ímpar, porém, faz-se necessário que essa inclusão ocorra de maneira efetiva e duradoura. O desenvolvimento de sistemas acessíveis para pessoas com deficiência visual tem crescido à medida que o uso dos smartphones aumenta em todos os segmentos da sociedade, bem como a criação de aplicativos móveis acessíveis. Como exemplos dessa tecnologia na promoção da saúde, destacam-se os aplicativos para auxiliar pessoas com deficiência visual a fazerem compras, locomoção nas cidades, consultas médicas e autocuidado (Ahmad \& Mozelius, 2019; Edemacu et al., 2019).

A utilização de aplicativos que auxiliem este grupo com deficiência visual pode evitar diversas complicações como episódios de hipoglicemia, assim como auxiliar com lembretes para consultas médicas e utilização de medicamentos, culminando em melhoria na acessibilidade desses pacientes aos cuidados em saúde (Jeffrey et al., 2019).

Com base no exposto e considerando o elevado índice de pessoas com acuidade visual reduzida por complicações do DM, surgiu a seguinte pergunta de pesquisa: como os pacientes com diabetes mellitus avaliam o uso de um aplicativo móvel com ênfase na promoção do autocuidado? Assim, o objetivo deste estudo foi avaliar a usabilidade de um aplicativo móvel para o autocuidado de pacientes com diabetes mellitus, idealizado por uma das autoras durante seu mestrado em gestão e inovação tecnológica em saúde.

\section{Metodologia}

Trata-se de um estudo metodológico, descritivo e de natureza aplicada. A pesquisa metodológica envolve o desenvolvimento, validação e avaliação de ferramentas e métodos de pesquisa (Polit \& Beck, 2018). A pesquisa aplicada tem por objetivo a geração de conhecimentos para aplicação prática para solucionar problemas específicos (Gerhardt \& Silveira, 2009).

O aplicativo, denominado DiaVision, foi desenvolvido a partir de um projeto de pesquisa em nível de mestrado de uma das autoras no Programa de Pós-Graduação em Gestão e Inovação Tecnológica em Saúde (PPGITS) da Universidade Federal de Sergipe (UFS), em parceria com a graduação em Engenharia da Computação da UFS.

O aplicativo foi desenvolvido segundo o modelo Systematic Design of Instruction (DIS), que é operacionalizado em quatro etapas: análise dos dados, design/desenvolvimento, implementação e avaliação da usabilidade (Falcade et al., 2016). Esse modelo de desenvolvimento de projetos é utilizado com o objetivo de nortear a equipe, desde a fase da ideia inicial do autor do projeto até a execução e fase de testes, considerando toda a linguagem de programação propriamente dita. 
A etapa de análise dos dados envolveu a elaboração do Documento de Visão para descrever as principais características do software desenvolvido, do mapeamento dos requisitos para avaliar os parâmetros mínimos necessários e do diagrama de use case que elencou as principais funcionalidades do aplicativo.

$\mathrm{Na}$ etapa de design/desenvolvimento foram definidos os aspectos para promover uma interação funcional e um bom aspecto visual do aplicativo, além de proporcionar acessibilidade ao usuário com deficiência visual. Em relação aos protótipos de tela, o DiaVision apresenta login, tela principal, configurações/perfil e diversas funcionalidades:

- Alimentação: apresenta uma lista das últimas alimentações registradas, opção para compartilhar registros e novo registro de alimentação (Figura 1).

Figura 1 - Tela da função alimentação do aplicativo DiaVision, Aracaju - SE, 2021.

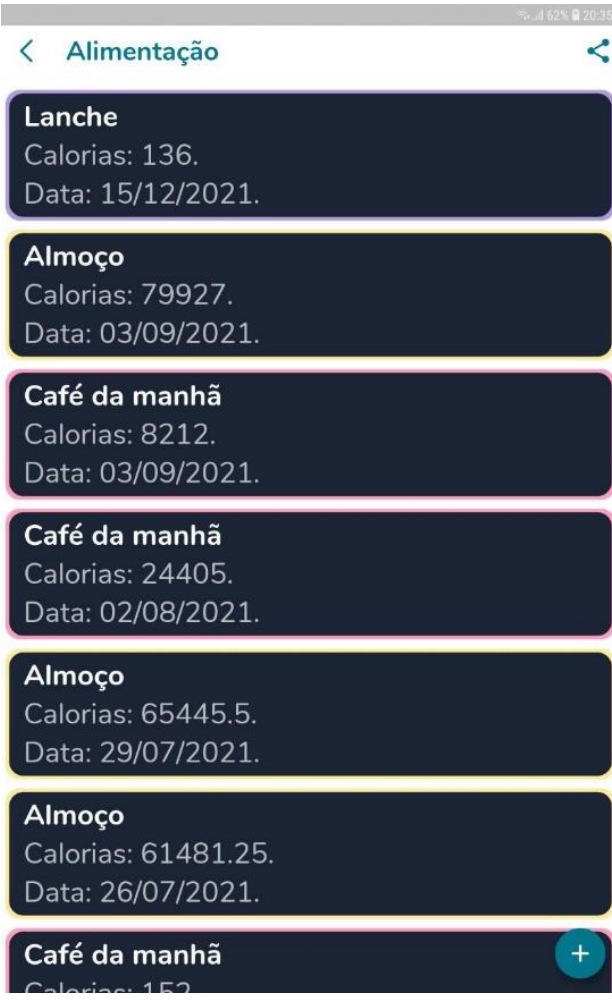

Fonte: DiaVision (2021).

- Aplicativos de visão: apresenta dicas de aplicativos acessíveis para pessoas com deficiência visual (Figura 2). 
Research, Society and Development, v. 11, n. 3, e1111326014, 2022

(CC BY 4.0) | ISSN 2525-3409 | DOI: http://dx.doi.org/10.33448/rsd-v11i3.26014

Figura 2 - Tela da função aplicativos de visão do DiaVision, Aracaju - SE, 2021.

W Apps de Visão
WeWALK
Descrição: WeWALK é um aplicativo de
navegação e exploração de acesso gratuito
desenvolvido por pessoas com deficiência
visual para pessoas com deficiência visual e
pessoas com baixa visão. Você pode obter
navegação passo a passo para onde deseja
ir e descobrir os locais ao seu redor de uma
maneira acessível.

Sullivan+
Descrição: O Sullivan + é um aplicativo de
auxílio visual para melhorar a acessibilidade
dos usuários com deficiência visual e com
baixa visão e informar aos usuários que
precisam de auxilio visual sobre as
informações percebidas pela câmera do
smartphone. Funções principais: modo Al,
reconhecimento de texto, reconhecimento
facial, descrição da imagem, reconhecimento
de cores, brilho da luz e lupa.

Emoções Deficiente Visual
Descrição: Este aplicativo permite que

Fonte: DiaVision (2021).

- Atividade física: apresenta lista de atividade física realizada, registro do tipo da atividade e sua duração (Figura 3).

Figura 3 - Tela da função atividade física do aplicativo DiaVision, Aracaju - SE, 2021.

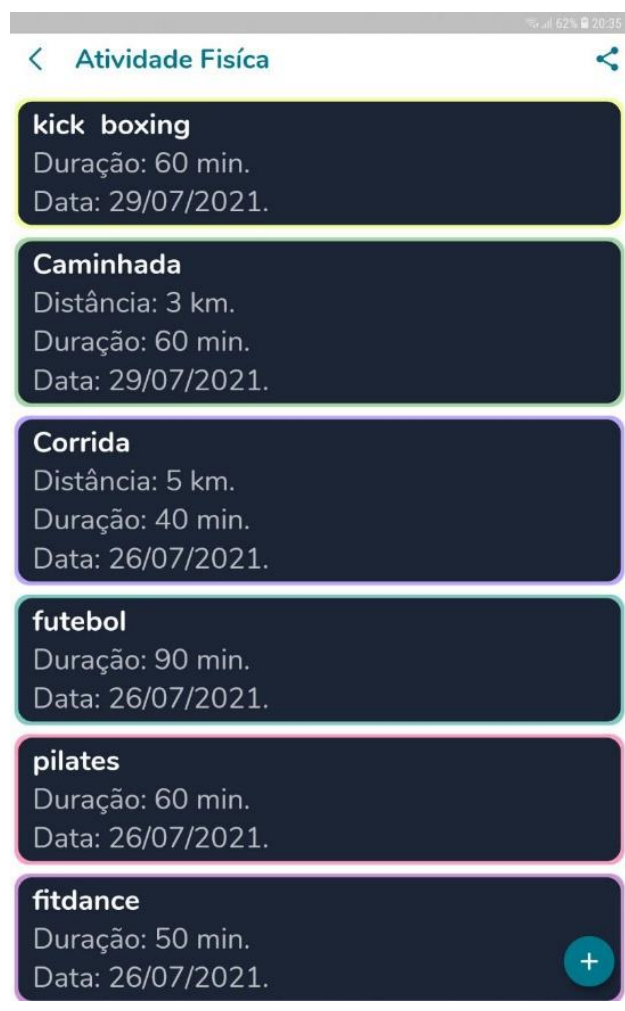

Fonte: DiaVision (2021). 
- Autocuidado: acesso a informações sobre autocuidado, como atividade física, controle e manutenção da glicemia, bons hábitos para manter a saúde dos rins, pés e visão, alimentação saudável, controle dos medicamentos e dicas gerais de saúde (Figura 4).

Figura 4 - Tela da função autocuidado do aplicativo DiaVision, Aracaju - SE, 2021.

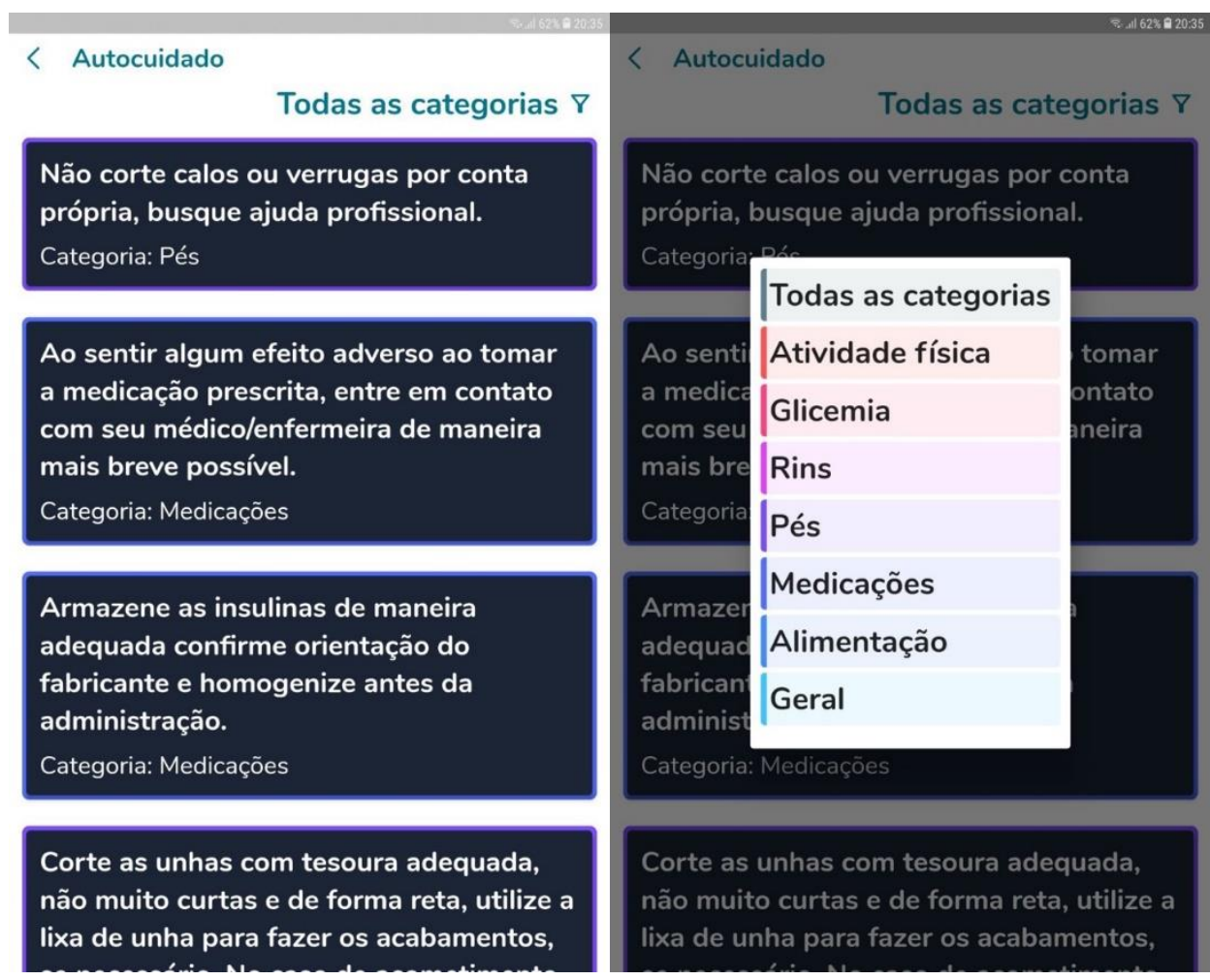

Fonte: DiaVision (2021).

- Centros de saúde: acesso ao nome, tipo, telefone, descrição, endereço e link do Google maps dos principais Centros de Saúde da cidade, desde centros de atenção primária até hospitais e clínicas terceirizadas (Figura 5). 
Research, Society and Development, v. 11, n. 3, e1111326014, 2022

(CC BY 4.0) | ISSN 2525-3409 | DOI: http://dx.doi.org/10.33448/rsd-v11i3.26014

Figura 5 - Tela da função centros de saúde do aplicativo DiaVision, Aracaju - SE, 2021.

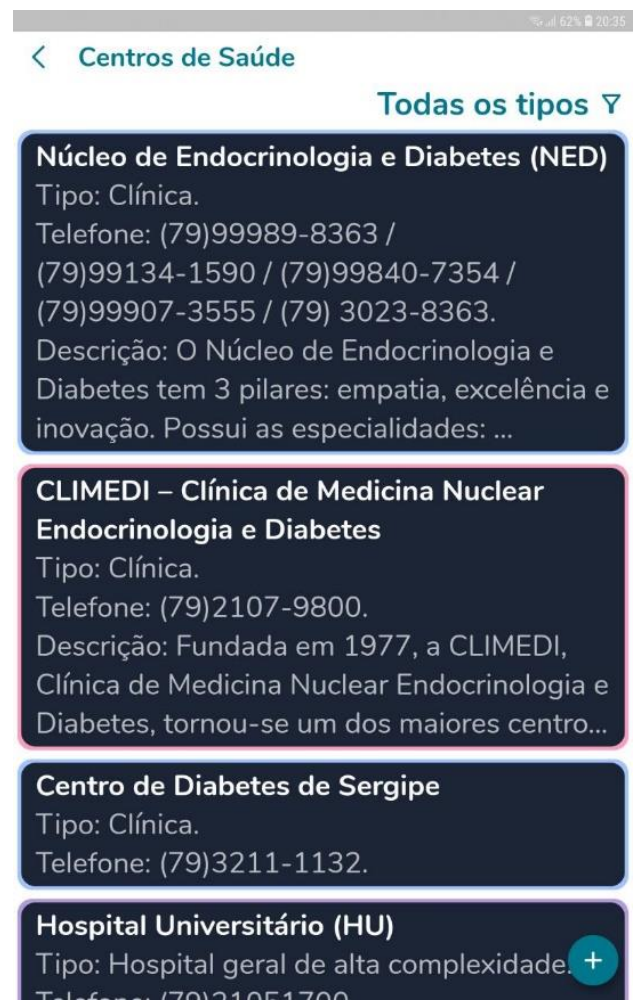

Fonte: DiaVision (2021).

- Glicemia: apresenta o histórico de glicemias registradas, edição de um registro, realização de novo registro, geração e compartilhamento de relatórios sobre as medidas registradas com avisos em casos hipo/hiperglicemia (Figura 6).

Figura 6 - Tela da função glicemia do aplicativo DiaVision, Aracaju - SE, 2021.

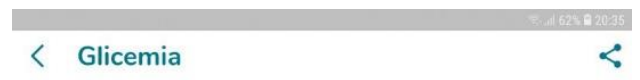

\section{Dia: 19/11/2021}

Valor (mg/dL): 380.

Horário: Pré refeição.

\section{Dia: $19 / 11 / 2021$}

Valor (mg/dL): 60 .

Horário: Em jejum.

\section{Dia: 06/10/2021}

Valor (mg/dL): 210 .

Horário: Pré refeição.

\section{Dia: 06/10/2021}

Valor (mg/dL): 320 .

\section{Dia: 06/10/2021}

Valor (mg/dL): 350.

Horário: Ao deitar.

\section{Dia: 03/09/2021}

Valor (mg/dL): 345.

Horário: 2 h pós refeição.

Fonte: DiaVision (2021). 
- Medicações: apresenta a opção de registrar todas as medicações utilizadas, assim como ter acesso e compartilhar a relação destas medicações consumidas (Figura 7).

Figura 7 - Tela da função medicações do aplicativo DiaVision, Aracaju - SE, 2021.
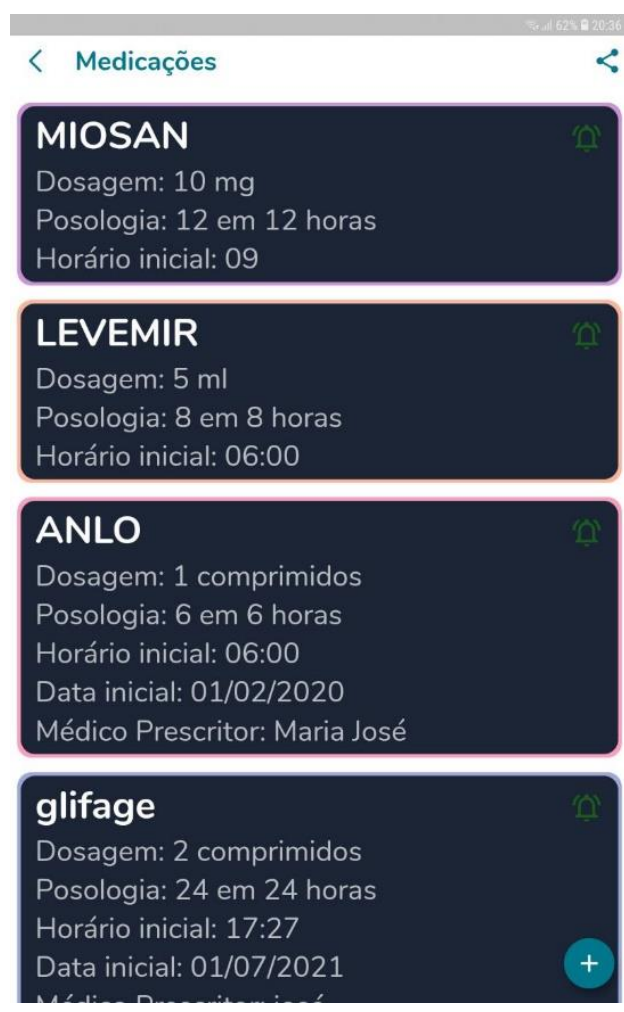

Fonte: DiaVision (2021).

- Pés: apresenta um roteiro para avaliação dos pés dos usuários, com perguntas sobre data da última consulta, temperatura da água que usa para lavar os pés, presença de hiperemia, calos e rachaduras, uso do protetor solar, checagem do sapato, hidratação, corte das unhas e higiene dos pés (Figura 8). 
Figura 8 - Tela da função pés do aplicativo DiaVision, Aracaju - SE, 2021.

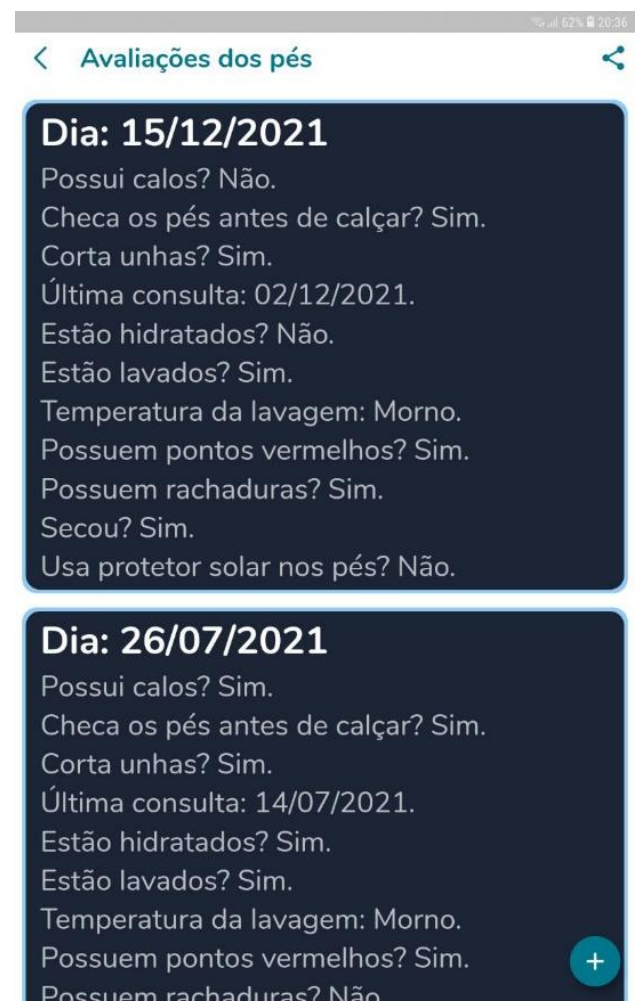

Fonte: DiaVision (2021)

- Rins: apresenta registro do volume, coloração e ardor da diurese, bem como gerar relatórios e encaminhamento das informações cadastradas (Figura 9).

Figura 9 - Tela da função rins do aplicativo DiaVision, Aracaju - SE, 2021.

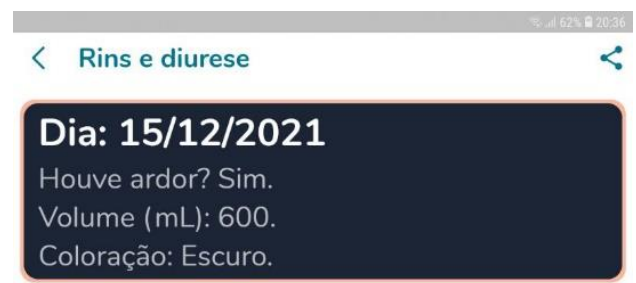

\section{Dia: $26 / 07 / 2021$}

Houve ardor? Não.

Volume (mL): 250 .

Coloração: Claro.

\section{Dia: $26 / 07 / 2021$}

Houve ardor? Sim.

Volume (mL): 150.

Coloração: Castanho.

\section{Dia: $21 / 07 / 2021$}

Houve ardor? Não.

Volume (mL): 60 .

Coloração: Claro. 
A etapa de implementação foi referente à entrada de dados, arquitetura do aplicativo, tradução para linguagem de programação, estruturação das funcionalidades e testes internos da aplicabilidade do protótipo. O Flutter foi o framework escolhido para programação do aplicativo através da linguagem de programação Dart para codificação da aplicação. Para a construção do Backend, utilizou-se o Back4app e o Parse Server para armazenamento de informações.

Esse estudo foca na descrição da quarta etapa referente à avaliação do dispositivo móvel. Para avaliação da usabilidade do aplicativo, a coleta de dados foi realizada em três ambulatórios na cidade de Aracaju/SE, localizados a saber: Hospital Universitário da UFS (HU-UFS), Hospital de Cirurgia (FBHC - Fundação de Beneficência Hospital de Cirurgia) e Centro Médico de Especialidades da prefeitura de Aracaju (CEMAR). A escolha desses locais justificou-se por serem considerados os principais ambulatórios públicos de referência no atendimento de pacientes com diabetes no Estado de Sergipe.

Foi realizado um teste piloto com 77 pacientes com a finalidade de avaliar a usabilidade do aplicativo. Os participantes do estudo foram pacientes diagnosticados com diabetes atendidos nos referidos ambulatórios. Os critérios de inclusão foram: possuir idade superior a 18 anos, possuir diabetes mellitus e apresentar habilidade cognitiva e psicomotora para manusear o tablet fornecido pela pesquisadora após explicação da funcionalidade do aplicativo. Os critérios de exclusão foram indivíduos com deficiência auditiva ou da fala pela necessidade de interação com o aplicativo através da ferramenta de auxílio auditivo para acessibilidade visual, assim como com a entrevistadora para receber as explicações do manuseio do aplicativo.

Os participantes foram selecionados de forma aleatória e não probabilística, com amostra por conveniência na sala de espera para atendimento nos referidos ambulatórios. Para o recrutamento, foram fornecidas as listas da agenda de atendimento do turno e os pacientes foram abordados pela entrevistadora em relação ao convite para participar da pesquisa. A coleta de dados ocorreu entre julho e setembro de 2021.

Os pacientes que se encontravam na sala de espera para atendimento foram abordados pela entrevistadora que explicou os objetivos da pesquisa e leu o Termo de Consentimento Livre e Esclarecido (TCLE). Após a concordância em participar da pesquisa e assinatura do TCLE, o usuário teve acesso ao aplicativo DiaVision no tablet da entrevistadora e, em seguida, procedeu à testagem do aplicativo por manuseio das diversas funções do aplicativo pelo próprio usuário portador de diabetes. Após finalizado o teste, os participantes responderam a perguntas de um formulário (Quadro 1) disponibilizado virtualmente através do Google forms. 
Quadro 1 - Formulário de avaliação da usabilidade do aplicativo DiaVision, Aracaju - SE, 2021.

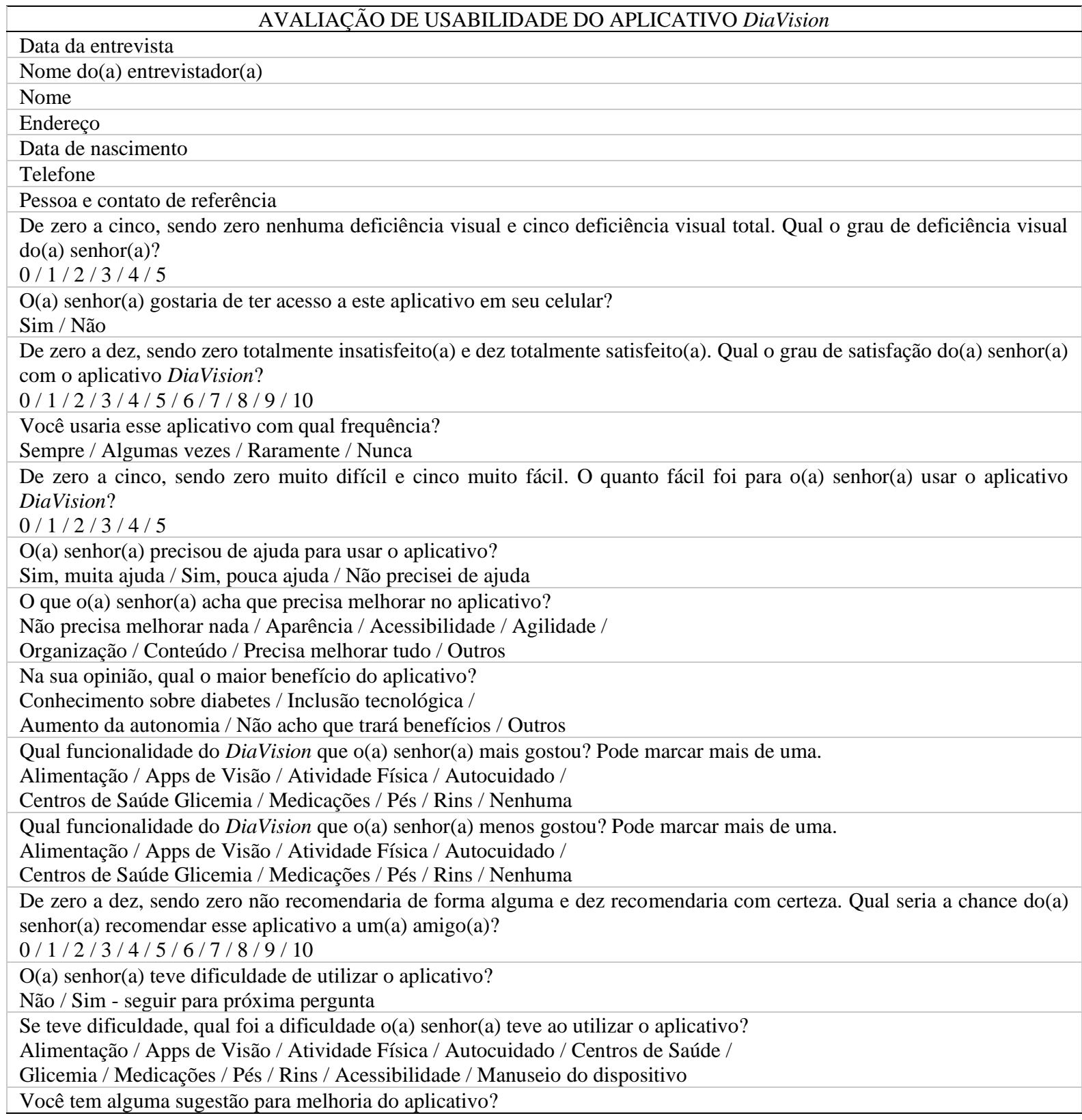

Fonte: Autores (2021).

Para análise dos resultados obtidos nas entrevistas, as variáveis categóricas, que expressam qualidade ou opinião dos participantes, foram descritas por meio de frequências absoluta e relativa percentual. As variáveis contínuas ou ordinárias, que expressam alguma medida numérica como idade ou os valores das escalas utilizadas no questionário utilizados, foram descritas por meio da moda e intervalo interquartil.

O teste de Shapiro-Wilk foi utilizado para avaliar a hipótese de aderência das variáveis contínuas a distribuição normal por ser o mais poderoso dentro da classe de testes para hipótese de aderência a distribuição normal (Razalli \& Yap, 2011). Como esta foi rejeitada em todos os testes efetuados, foram utilizados os Testes de Mann-Whitney (dois grupos de variáveis) e KruskalWallis (três ou mais grupos de variáveis) para avaliar a hipótese de igualdade de moda por serem os mais indicados para esta análise estatística.

A correlação entre variáveis ordinais utilizadas na pesquisa e relacionadas no quadro 1 foi testada por meio da correlação de Spearman e a hipótese de ausência de correlação foi testada devido a superioridade em poder deste teste no cenário do estudo 
apresentado e descrita nos resultados. O nível de significância adotado em todo o estudo foi de $5 \%$ e o software utilizado foi o R Core Team 2021 (versão 4.1.0).

Este estudo foi submetido ao Comitê de Ética em Pesquisa da UFS (CEP-UFS) e aprovado sob o número CAE 33220620.3.0000.5546, com autorização para a coleta de dados. Foram respeitados os critérios da Resolução 466/2012, do Conselho Nacional de Saúde.

\section{Resultados e Discussão}

Foram incluídos 77 pacientes portadores de diabetes atendidos nos referidos ambulatórios. Dentre os participantes da pesquisa, 54 eram do sexo feminino, correspondendo a 70,1\% da amostra. A idade variou entre 18 e 83 anos, com média de 53,1 anos. Todos eram habitantes de munícipios de Sergipe e regularmente cadastrados como pacientes. Essa média de idade foi semelhante ao encontrado por Julian et al. (2020), que produziram revisão dos custos das complicações macrovasculares em pessoas com diabetes utilizando dados do DATASUS (sistema de dados do sistema público de saúde) em que a média encontrada foi de 59,5. A maior prevalência portadoras de diabetes do sexo feminino também foi verificada no estudo realizado por Rezende et al. (2015) que avaliaram 1.076 pacientes sergipanos, sendo 63,5\% destes do sexo feminino, por coleta de dados pessoais e protocolos clínicos para estratificação do risco de pé diabético. Portanto, a amostra do DiaVision encontra semelhança na literatura com outras coortes com público semelhante, o que garante o seu poder de comparação.

Em relação à deficiência visual, em escala de zero (0) a cinco (5) em que zero (0) significa ausência de comprometimento da acuidade visual e cinco (5) significa deficiência visual total, foi identificada uma moda igual a três (3). Dentre os participantes, 2,6\% afirmaram apresentar deficiência visual total (Figura 1). Estes participantes com deficiência visual total usaram o aplicativo com auxílio da pesquisadora responsável.

Em revisão sistemática produzida por Leasher et al. (2016), em que foram avaliados dados publicados entre 1990 e 2010, foi encontrada prevalência estimada em 2,5\% de deficiência visual total devido a retinopatia diabética quando analisados todos os pacientes com deficiência total por todas as causas. Verifica-se, portanto, que a retinopatia é uma causa importante de deficiência visual total entre os pacientes portadores de diabetes, fato que reflete a necessidade de promover uma melhor acessibilidade a esse público.

Figura 10 - Graus de deficiência visual dos pacientes com Diabetes Mellitus, Aracaju - SE, 2021.

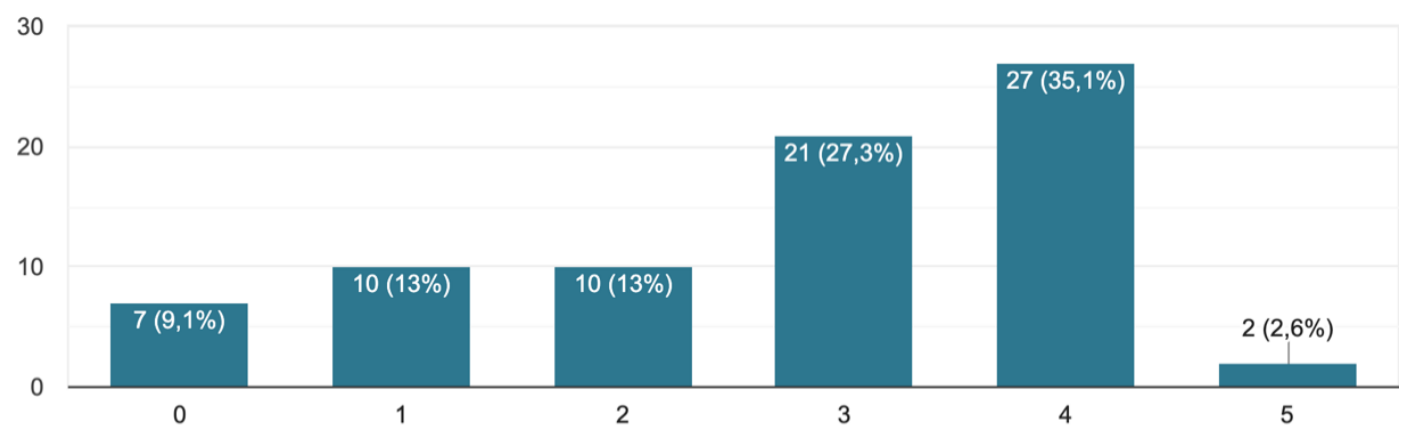

Fonte: Autores (2021).

A avaliação da usabilidade do DiaVision demonstrou que o déficit visual total fazia parte da minoria de pacientes. Semelhantemente, Hazari et al. (2020), ao validarem um aplicativo para pacientes com retinopatia diabética, identificaram que a maioria dos testados não apresentava alto grau de deficiência visual. Dado semelhante encontrado no estudo de usabilidade do Diavision em que 2,6\% afirmaram ter deficiência visual total. 
Ao serem questionados sobre o desejo de possuir o aplicativo DiaVision no dispositivo móvel pessoal, 90,1\% dos participantes afirmaram positivamente que gostariam de ter. O grau de satisfação geral dos participantes quanto o aplicativo foi avaliado em uma escala de zero (0) a dez (10), em que zero (0) significa insatisfação e dez (10) significa satisfação máxima. Observou-se que a avaliação geral dos usuários variou entre cinco (5) e dez (10) com média de 9,3 (Figura 2).

Figura 11 - Grau de satisfação geral dos participantes quanto ao aplicativo DiaVision, Aracaju - SE, 2021.

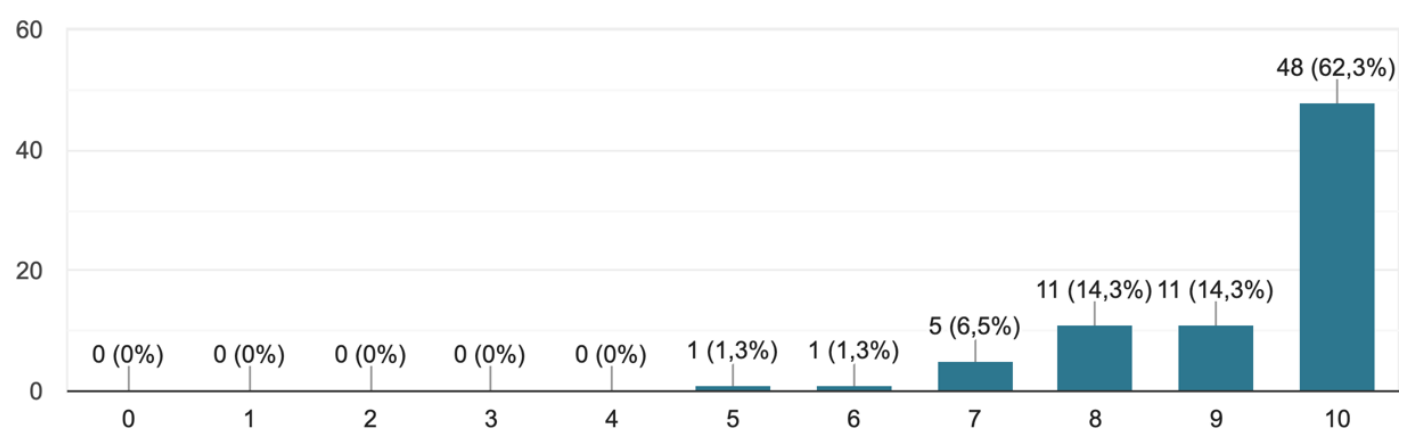

Fonte: Autores (2021).

Para que uma ferramenta seja útil no manejo de patologias crônicas, é importante que esta seja acessível para ser usada com a maior frequência possível. Neste sentido, a frequência de uso foi questionada, de forma que $53,2 \%$ dos participantes responderam que usariam o aplicativo sempre, $31,2 \%$ relataram que usariam as vezes, $14,3 \%$ raramente e 1,3\% nunca usariam. Kumar et al. (2018) verificaram que $25 \%$ dos pacientes usariam aplicativo em que o paciente ou cuidador era treinado por um pesquisador com experiência em tratar diabetes. O resultado foi uma redução significativa dos índices de hemoglobina glicada. O estudo de usabilidade do DiaVision encontrou frequência maior de uso. Isso pode se dever ao caráter educacional e voltado para o autocuidado que o DiaVision tem quando comprado com outros aplicativos voltados para diabetes.

Ressalta-se que 51,95\% dos participantes afirmaram sentir dificuldades em manusear o aplicativo. Dentre estes usuários, $59,9 \%$ afirmaram que a maior dificuldade foi no item alimentação $(\mathrm{p}=0,885)$. Tendo em vista que o paciente portador de diabetes, principalmente do tipo 2, apresenta excesso de peso simultâneo, devido aos maus hábitos alimentares associados a baixa adesão a atividade física, é esperado que qualquer intervenção que tente gerar impacto na mudança de maus hábitos arraigados no cotidiano desses pacientes não seja bem aceita.

Alonso-Domínguez et al. (2017) desenvolveram estudo em que os participantes de um ambulatório universitário foram divididos em dois grupos: um grupo controle e outro grupo intervenção. O grupo intervenção além das orientações especificas sobre a dieta mediterrânea, receberam auxílio de um aplicativo para smartphone por 12 semanas e foram estimulados a participar de um programa de caminhada por cinco semanas seguidas. Ao final do estudo, o grupo intervenção que utilizou a ferramenta virtual apresentou melhores resultados, inclusive na melhoria dos hábitos alimentares. Portanto, apesar de o teste de usabilidade demonstrar uma tendência a baixa adesão no quesito alimentação, entende-se que, com o auxílio e incentivo para utilização do aplicativo, os pacientes possam se engajar mais em seu tratamento e aderir a melhores hábitos alimentares.

Questionou-se aos usuários a necessidade de melhorias no aplicativo, do total de entrevistados, 45,5\% dos usuários afirmaram não haver necessidade de melhorias. Dos usuários que consideraram a necessidade de melhorias, 35,1\% sugeriram ajustes quanto à acessibilidade do aplicativo (Figura 3). 
Figura 12 - Avaliação dos participantes quanto à necessidade de melhorias do aplicativo DiaVision, Aracaju - SE, 2021.

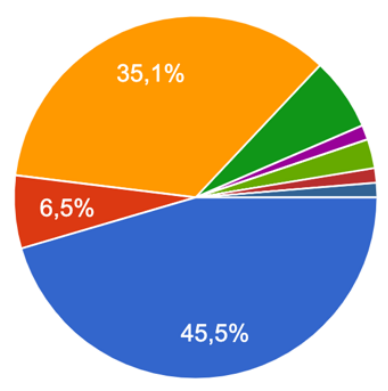

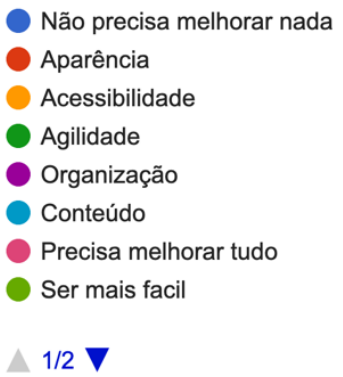

Fonte: Autores (2021).

Acessibilidade, neste contexto, se referiu à facilidade que o usuário encontra para acessar o aplicativo, dada sua condição de deficiência visual. Assim, a acessibilidade é condição fundamental para o sucesso do aplicativo. Whitehead et al. (2016) conduziram uma revisão sobre a utilização de aplicativos para manejo de doenças crônicas e destacaram que a acessibilidade ao software é uma condição imprescindível para que o aplicativo possa ser uma ferramenta de melhoria no autocuidado dos pacientes. Os pacientes entrevistados para avaliar a usabilidade do DiaVision referiram em 23,4\% das respostas que não precisariam de nenhuma ajuda para usar o aplicativo, enquanto 40,3\% referiram apresentar pouca dificuldade. Portanto, para $63,7 \%$ dos entrevistados, o DiaVision foi considerado um aplicativo fácil de ser manuseado.

Quanto ao questionamento sobre a maior contribuição do aplicativo em relação aos benefícios proporcionados aos participantes, 76,6\% declararam que o DiaVision contribui para o conhecimento sobre o diabetes. O conhecimento adequado da própria doença é passo de alta relevância para o sucesso do autocuidado de pacientes portadores de diabetes. Jeffrey et al. (2019) demonstraram, em pesquisa que incluiu aplicativos para diabetes, que os maiores impactos positivos são gerados quando os aplicativos unem ferramentas de boa qualidade tecnológica com ferramentas que agreguem conhecimento para o paciente. Assim o DiaVision demonstrou gerar maior aceitação por parte do usuário por valorizar a parte educacional do cuidado do paciente portador de diabetes.

Em relação à funcionalidade do DiaVision que mais agradou os participantes, 67,5\% dos usuários afirmaram que foi o registro dos valores de glicemia. Yu et al. (2019), em estudo com quatro grupos, demonstraram que a combinação do uso de aplicativo móvel para diabetes e automonitorização de glicemia gerou impacto significativo na melhoria dos parâmetros de hemoglobina glicada, dentre outros desfechos. O DiaVision apresenta, portanto, alta probabilidade de gerar impacto positivo no cuidado destes pacientes exatamente por incluir essa ferramenta citado pelos voluntários como uma das maiores contribuições do aplicativo.

Foi questionado aos usuários a possibilidade de recomendação do aplicativo a outros pacientes por meio de uma escala de zero (0) a dez (10), em que zero (0) significa nenhuma recomendação e dez (10) significa recomendação máxima. A maioria $(70,1 \%)$ dos usuários respondeu que faria recomendação máxima a outros pacientes (Figura 4). 
Figura 13 - Opinião dos participantes quanto à possibilidade de recomendação do aplicativo DiaVision a outros pacientes, Aracaju - SE, 2021.

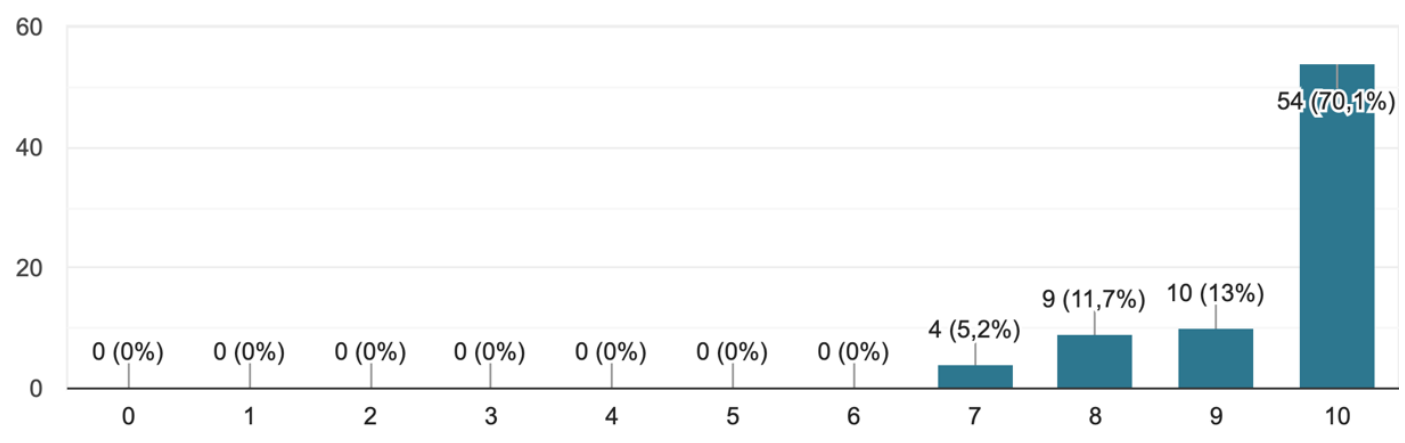

Fonte: Autores (2021).

A utilização de softwares para dispositivos móveis tem se destacado como importante ferramenta de apoio no tratamento de pacientes portadores de diabetes. A adesão ao uso do aplicativo passa diretamente pelo entendimento de usabilidade pelo usuário. O DiaVision obteve um elevado índice de aprovação, de forma que 90,9\% afirmaram que gostariam de ter acesso ao aplicativo no próprio celular e apresentou uma médica de recomendação de 9,5 por parte dos usuários durante a testagem do aplicativo. Índice de aprovação similar a Kumar et al. (2018), que encontraram em sua pesquisa um índice de $94 \%$ de aceitação por parte dos participantes, informando que o aplicativo melhorou o grau de cuidado com o diabetes.

A Tabela 1 reúne todas as respostas de acordo com os questionamentos na avaliação do aplicativo DiaVision aos participantes.

Tabela 1 - Frequências absolutas e relativas, média e moda dos dados da avaliação do aplicativo DiaVision pelos participantes, Aracaju - SE, 2021.

\begin{tabular}{|c|c|c|c|c|}
\hline & n & $\%$ & Média (DP) & $\begin{array}{l}\text { Moda } \\
\text { (IIQ) }\end{array}$ \\
\hline Idade & & & $53,1(16,5)$ & $54,9(39,3-62,4)$ \\
\hline Sexo & & & & \\
\hline Feminino & 54 & 70,1 & & \\
\hline Masculino & 23 & 29,9 & & \\
\hline Qual o grau de deficiência visual do(a) senhor(a)? & & & $2,7(1,4)$ & $3(2-4)$ \\
\hline O(a) senhor(a) gostaria de ter acesso a este aplicativo em seu celular? & & & & \\
\hline $\operatorname{Sim}$ & 70 & 90,9 & & \\
\hline Não & 7 & 9,1 & & \\
\hline $\begin{array}{l}\text { Qual o grau de satisfação do(a) senhor(a) com o aplicativo DiaVision? } \\
\text { Você usaria esse aplicativo com qual frequência? }\end{array}$ & & & $9,3(1,1)$ & $10(9-10)$ \\
\hline Sempre & 41 & 53,2 & & \\
\hline Algumas vezes & 24 & 31,2 & & \\
\hline Raramente & 11 & 14,3 & & \\
\hline Nunca & 1 & 1,3 & & \\
\hline $\begin{array}{l}\text { O quanto fácil foi para o(a) senhor(a) usar o aplicativo Dia Vision? } \\
\text { O(a) senhor(a) precisou de ajuda para usar o aplicativo? }\end{array}$ & & & $4,0(1,2)$ & $4(3-5)$ \\
\hline Sim, muita ajuda & 28 & 36,4 & & \\
\hline Sim, pouca ajuda & 31 & 40,3 & & \\
\hline Não precisei de ajuda & 18 & 23,4 & & \\
\hline O que o(a) senhor(a) acha que precisa melhorar no aplicativo? & & & & \\
\hline Não precisa melhorar & 35 & 45,5 & & \\
\hline Aparência & 5 & 6,5 & & \\
\hline Acessibilidade & 27 & 35,1 & & \\
\hline Agilidade & 5 & 6,5 & & \\
\hline Organização & 1 & 1,3 & & \\
\hline
\end{tabular}


Outros

Na sua opinião, qual o maior benefício do aplicativo?

Conhecimento sobre diabetes

Inclusão tecnológica

Aumento da autonomia

Qual funcionalidade do DiaVision que o(a) senhor(a) mais gostou?

Alimentação

Apps de Visão

Ativida de Física

Autocuidado

Centros de Saúde

Glicemia

Medicações

Pés

Rins

Qual funcionalidade do DiaVision que o(a) senhor(a) mais gostou?

Alimentação

Apps de Visão

Atividade Física

Autocuidado

Centros de Saúde

Glicemia

Medicações

Pés

Rins

Nenhuma

Qual seria a chance do(a) $\operatorname{senhor}(a)$

recomendar esse aplicativo a um(a) amigo(a)?

$\mathbf{O}(\mathbf{a})$ senhor(a) teve dificuldade de utilizar o aplicativo?

Sim

Não

Se teve dificuldade, qual foi a dificuldade o(a)

senhor(a) teve ao utilizar o aplicativo?

Acessibilidade

Alimentação

Manuseio do dispositivo
$4 \quad 5,2$

5976,6

$8 \quad 10,4$

1013,0

$30 \quad 39,0$

1114,3

$11 \quad 14,3$

$17 \quad 22,1$

$4 \quad 5,2$

$52 \quad 67,5$

$22 \quad 28,6$

$\begin{array}{ll}23 & 29,9\end{array}$

1013,0

$22 \quad 28,6$

1722,1

$21 \quad 27,3$

33,9

22,6

$0 \quad 0,0$

45,2

$\begin{array}{ll}7 & 9,1\end{array}$

79,1

1013,0

$9,5(0,9) \quad 10(9-10)$

$40 \quad 51,9$

3748,1

25,1

2359,0

$14 \quad 35,9$

Legenda: $\mathrm{n}$ - frequência absoluta. \% - frequência relativa percentual. DP - Desvio Padrão. IIQ - Intervalo Interquartil. Fonte: Autoras (2021).

Os dados comparativos e seus respectivos níveis de significância estão demonstrados na Tabela 2.

Tabela 2 - Dados comparativos e seus respectivos níveis de significância referentes à avaliação do aplicativo DiaVision pelos participantes, Aracaju, 2021.

\begin{tabular}{|c|c|c|c|}
\hline & $\begin{array}{l}\text { Média } \\
\text { (DP) }\end{array}$ & $\begin{array}{r}\text { Moda } \\
(\text { IIQ) }\end{array}$ & p-valor \\
\hline \multicolumn{4}{|l|}{ Sexo } \\
\hline Feminino & $2,8(1,3)$ & $3(2-4)$ & $1,000^{\mathrm{M}}$ \\
\hline Masculino & $2,7(1,5)$ & $3(2-4)$ & \\
\hline \multicolumn{4}{|c|}{$\begin{array}{l}\text { O(a) senhor(a) gostaria de ter acesso a } \\
\text { este aplicativo em seu celular? }\end{array}$} \\
\hline $\operatorname{Sim}$ & $2,8(1,4)$ & $3(2-4)$ & $0,539^{\mathrm{M}}$ \\
\hline Não & $2,6(1,1)$ & $3(2-4)$ & \\
\hline \multicolumn{4}{|c|}{ Você usaria esse aplicativo com qual frequência? } \\
\hline Sempre & $2,7(1,5)$ & $3(1-4)$ & $0,597^{\mathrm{K}}$ \\
\hline Algumas vezes & $3(1,1)$ & $3(2,5-4)$ & \\
\hline Raramente & $2,5(1,6)$ & $3(1,5-4)$ & \\
\hline
\end{tabular}

O(a) senhor(a) precisou de ajuda para

usar o aplicativo? 
Sim, muita ajuda

Sim, pouca ajuda

Não precisei de ajuda

O que o(a) senhor(a) acha que precisa

melhorar no aplicativo?

Não precisa melhorar

Acessibilidade

Agilidade

Outros

Na sua opinião, qual o maior benefício do aplicativo?

Conhecimento sobre diabetes

Inclusão tecnológica

Aumento da autonomia

Qual funcionalidade do DiaVision que

o(a) senhor(a) mais gostou?

Alimentação

Sim

Não

Apps de Visão

Sim

Não

Atividade Física

Sim

Não

Autocuidado

Sim

Não

Centros de Saúde

Sim

Não

Glicemia

Sim

Não

Medicações

Sim

Não

Pés

Sim

Não

Rins

Sim

Não

Qual funcionalidade do DiaVision que o(a)

senhor(a) mais gostou?

\section{Alimentação}

Sim

Não

Apps de Visão

Sim

Não

Atividade Física

Sim

Não

Autocuidado

Sim

Não

Medicações

Sim

Não

Pés

Sim

$\begin{array}{lll}3,5(1)^{\mathrm{a}} & 4(3-4) & 0,002^{\mathrm{K}} \\ 2,3(1,4)^{\mathrm{b}} & 3(1-3) & \\ 2,4(1,4)^{\mathrm{b}} & 3(1-4) & \\ & & \\ 2,2(1,3)^{\mathrm{a}} & 2(1-3) & 0,012^{\mathrm{K}} \\ 3,2(1,4)^{\mathrm{b}} & 4(3-4) & \\ 2,4(1,3)^{\mathrm{a}, \mathrm{b}} & 3(1-3) & \\ 3(0,8)^{\mathrm{a}, \mathrm{b}} & 3(2,5-3,5) & \\ & & \\ 2,7(1,4) & 3(2-4) & 0,400^{\mathrm{K}} \\ 3,3(1) & 3,5(3-4) & \\ 2,7(1,3) & 3(1-4) & \end{array}$

$2,4(1,5)$

$2,9(1,3)$

2,5 (1-4)

$3(2,5-4)$

$2,6(1,7)$

$2,8(1,3)$

$3(1,5-4)$

3 (2-4)

2,3 (1,3)

$2,8(1,4)$

$2(1,5-4)$

3 (2-4)

$2,4(1,5)$

$3(1-4)$

$3(2-4)$

$2(1,8)$

$2,8(1,3)$

$2(0,5-3,5)$

3 (2-4)

$2,9(1,2)$

$2,4(1,7)$

$3(2-4)$

3 (1-4)

$3(1,4)$

3 (3-4)

$2,7(1,4)$

3 (2-4)

$3,3(1,2)$

$4(3-4)$

$2,5(1,4)$

$3(1-4)$

$3,4(0,8)$

$2,6(1,4)$

$4(3-4)$

$3(1,5-4)$

$3,5(1)$

$2,5(1,4)$

4 (3-4)

$3(1-3,5)$

$2,5(1,5)$

$3(1-4)$

$2,8(1,3)$

3 (2-4)

$3,2(1,2)$

$2,6(1,4)$

4 (3-4)

3 (1-4)

$4,7(0,6)$

$2,7(1,3)$

$5(4,5-5)$

3 (2-4)

$1,8(1,3)$

$2(1-2,5)$

3 (2-4)

$2(1,3)$

$3(1-3)$

$0,093^{\mathrm{M}}$ 


\begin{tabular}{|c|c|c|c|}
\hline Não & $2,8(1,4)$ & $3(2-4)$ & \\
\hline \multicolumn{4}{|l|}{ Rins } \\
\hline Sim & $2,3(1,3)$ & $2(1,5-3)$ & \multirow[t]{2}{*}{$0,304^{\mathrm{M}}$} \\
\hline Não & $2,8(1,4)$ & $3(2-4)$ & \\
\hline \multicolumn{4}{|l|}{ Nenhuma } \\
\hline Sim & $2,2(1,1)$ & $2,5(1-3)$ & \multirow[t]{2}{*}{$0,106^{\mathrm{M}}$} \\
\hline Não & $2,8(1,4)$ & $3(2-4)$ & \\
\hline \multicolumn{4}{|c|}{$\begin{array}{l}\text { O(a) senhor(a) teve dificuldade de } \\
\text { utilizar o aplicativo? }\end{array}$} \\
\hline $\operatorname{Sim}$ & $3,1(1,2)$ & $3(2-4)$ & \multirow[t]{2}{*}{$0,022^{\mathrm{M}}$} \\
\hline Não & $2,4(1,4)$ & $3(1-4)$ & \\
\hline \multicolumn{4}{|c|}{$\begin{array}{l}\text { Se teve dificuldade, qual foi a dificuldade } \\
\text { o(a) senhor(a) teve ao utilizar o aplicativo? }\end{array}$} \\
\hline Acessibilidade & $3,5(0,7)$ & $3,5(3-4)$ & \multirow[t]{3}{*}{$0,885^{\mathrm{K}}$} \\
\hline Alimentação & $3(1,4)$ & $3(2-4)$ & \\
\hline Manuseio do dispositivo & $3,1(1)$ & $3(3-4)$ & \\
\hline
\end{tabular}

Legenda: DP - Desvio Padrão. IIQ - Intervalo Interquartil. M - Teste de Mann-Whitney. K - Teste de Kruskal-Wallis. a,b Subgrupos distintos para o teste de Mann-Whitney-Dunn. Fonte: Autoras (2021).

Realizou-se uma análise comparativa sobre a hipótese de que o grau de deficiência visual, assim como a idade, poderia interferir na satisfação quanto à usabilidade do aplicativo (Tabelas 3 e 4). Observou-se que idade interferiu na facilidade no uso do aplicativo, como também no índice de recomendação do aplicativo ( $p<0,001$ e p=0,007, respectivamente). Quanto ao grau de deficiência visual, houve significância estatística em relação ao grau de satisfação e grau de recomendação do aplicativo (p=0,020 e $\mathrm{p}=0,020$, respectivamente).

Tabela 3 - Relação entre o grau de deficiência visual e a satisfação quanto a usabilidade do aplicativo DiaVision, Aracaju - SE, 2021.

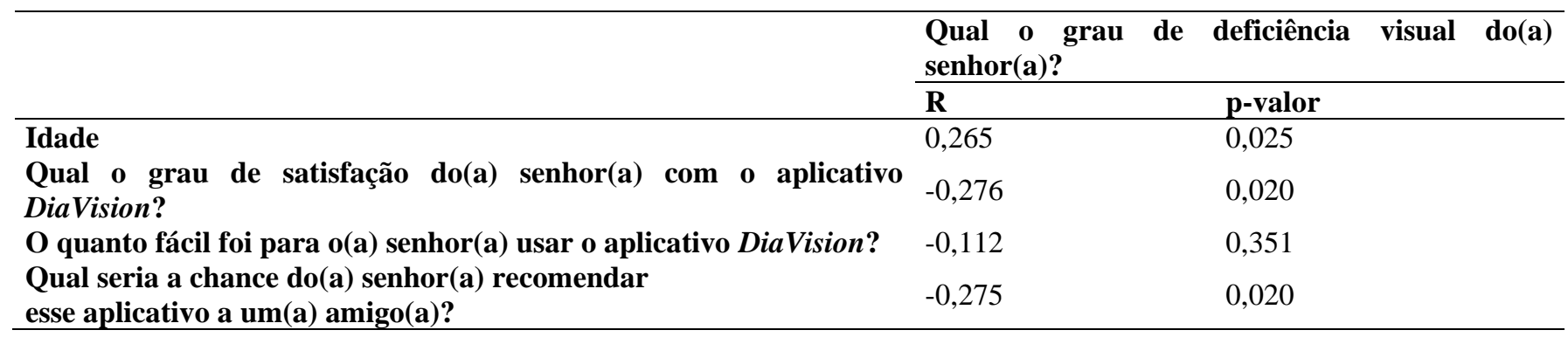

Legenda: R - Correlação de Spearman. Fonte: Autoras (2021).

Tabela 4 - Relação entre a idade e a satisfação quanto a usabilidade do aplicativo DiaVision, Aracaju - SE, 2021.

\begin{tabular}{lcc}
\hline & Idade & \multicolumn{1}{c}{ p-valor } \\
\cline { 2 - 2 } Qual o grau de satisfação do(a) senhor(a) com o aplicativo DiaVision? & $-0,204$ & 0,088 \\
O quanto fácil foi para, o(a) senhor(a) usar o aplicativo Dia Vision? & $-0,569$ & $<0,001$ \\
Qual seria a chance do(a) senhor(a) recomendar esse aplicativo a um(a) amigo(a)? & $-0,319$ & 0,007 \\
\hline
\end{tabular}

Legenda: R - Correlação de Spearman. Fonte: Autoras (2021).

Os resultados demonstram que o grau de deficiência visual e a idade dos pacientes interferem na satisfação e consequente recomendação do aplicativo. Resultado semelhante foi encontrado por Bilong et al. (2019), em que os autores demonstraram a validação de diagnóstico de retinopatia diabética por meio de fotografia retiniana através de smartphone. Os usuários com maior grau de deficiência visual aderiram menos ao protocolo e, portanto, obtiveram resultados piores.

Vale ressaltar que o DiaVision não se propõe a auxiliar o diagnóstico de retinopatia diabética, mas os pacientes com maior grau de deficiência visual também demonstram maior dificuldade na utilização das ferramentas do aplicativao, o que é 
mais do que esperado nesses casos. Dessa forma, o ideal é que aplicativos possam ser utilizados em fases iniciais da retinopatia diabética, tendo em vista que serão operacionalizados da melhor forma, como também poderão gerar melhor controle dos níveis glicêmicos e reduzir as complicações da retinopatia.

\section{Conclusão}

A etapa de avaliação da usabilidade foi realizada com pacientes portadores de diabetes mellitus atendidos em ambulatórios através de um formulário. A maioria dos pacientes avaliou o DiaVision com um elevado nível de satisfação e informou que gostaria de ter o aplicativo. A dificuldade ao usar o aplicativo mais frequente foi relacionada à funcionalidade da alimentação e a principal sugestão de melhoria do aplicativo foi quanto à acessibilidade. A funcionalidade do DiaVision que mais agradou os participantes foi o registro dos valores de glicemia. A idade e o grau de deficiência visual interferiram na facilidade no uso e no índice de recomendação do aplicativo.

As limitações encontradas para o desenvolvimento desta pesquisa foram a dificuldade de manipulação do tablet por parte dos potenciais usuários e restrição de acesso físico aos participantes da pesquisa devido ao isolamento social vivenciado durante a pandemia da COVID-19, além do reduzido número de participantes para avaliação de usabilidade e a falta de validação do aplicativo desenvolvido. Além disso, os usuários testaram o aplicativo através do tablet da pesquisadora que, por se tratar de um aparelho com maiores dimensões, pode gerar um viés em relação à questão da acessibilidade, tendo em vista que os pacientes com diabetes poderão usar o aplicativo em dispositivos móveis de menor tamanho com consequente diminuição das imagens reproduzidas na tela.

Como perspectivas futuras, espera-se que o DiaVision possa ser ampliado a nível nacional e internacional, tanto em sua base de dados, quanto em caráter tecnológico, acrescentando mais ferramentas como inserção da calculadora de insulina e rede de apoio entre os usuários. Ademais, outros ajustes poderão ser realizados como: otimização das ferramentas existentes com análise dos dados fornecidos nas avaliações dos pés e rins, fornecimento dos dados para os usuários em formato de gráficos, ampliação das funcionalidades da atividade física, aprimoramento do layout das telas e resultados das anotações dos usuários, aumento da acessibilidade para atender às necessidades dos usuários com deficiência visual maior. Sugere-se o desenvolvimento de mais estudos sobre a usabilidade do DiaVision com amostra maior, validação da ferramenta e avaliação do impacto do aplicativo no processo educacional dos pacientes com diabetes.

\section{Referências}

Ahmad, A. \& Mozelius, P. (2019). Critical factors for human computer interaction of eHealth for older adults. ACM Digital Library, 58-62.

Alonso-Domínguez, R. et al. (2019). Effectiveness of A Multifactorial Intervention in Increasing Adherence to the Mediterranean Diet among Patients with Diabetes Mellitus Type 2: A Controlled and Randomized Study (EMID Study). Nutrients, 11(1), 162.

Bilong, Y. et al. (2019). Validation of Smartphone-Based Retinal Photography for Diabetic Retinopathy Screening. Ophthalmic Surg Lasers Imaging Retina, $50,18-22$.

Birkhoff, S. D. \& Smeltzer, S. C. (2017). Perceptions of Smartphone User-Centered Mobile Health Tracking Apps Across Various Chronic Illness Populations: An Integrative Review. J Nurs Scholarsh, 49(4), 371-8.

Bourne, R. R. A. et al. (2021a). Causes of blindness and vision impairment in 2020 and trends over 30 years, and prevalence of avoidable blindness in relation to VISION 2020: the Right to Sight: an analysis for the Global Burden of Disease Study. Lancet Glob Health, 9(2), 144-60.

Bourne, R. R. A. et al. (2021b). Trends in prevalence of blindness and distance and near vision impairment over 30 years: An analysis for the Global Burden of Disease Study. Lancet Glob Health, 9(2), 130-43.

Chrvala, C. A., Sherr, D., \& Lipman, R. D. (2016). Diabetes self-management education for adults with type 2 diabetes mellitus: A systematic review of the effect on glycemic control. Patient Education and Counseling, 99(6), 926-43.

Edemacu, K. et al. (2019). Privacy Provision in Collaborative Ehealth with Attribute-Based Encryption: Survey, Challenges and Future Directions. IEEE Access, 7, 89614-36. 
Falcade, A. et al. (2016). Design Instrucional: um comparativo de metodologias para definição de abordagem em mundo virtual. Anais do Congresso Brasileiro de Informática na Educação, Uberlândia, MG, Brasil, 5.

Gerhardt, T. E. \& Silveira, D. T. (2009). Métodos de pesquisa. Porto Alegre: Editora da UFRGS.

Hazari, H. et al. (2020). Validation of the visual acuity iPad app Eye Chart Pro compared to the standard Early Treatment Diabetic Retinopathy Study chart in a low-vision population. $J$ Telemed Telecare, $0(0), 1-7$.

International Diabetes Federation. (2019). IDF Diabetes Atlas: Projections of the prevalence of hyperglycaemia in pregnancy in 2019 and beyond: Results from the International Diabetes Federation Diabetes Atlas. International Diabetes Federation.

Jeffrey, B. et al. (2019). Mobile phone applications and their use in the self-management of Type 2 Diabetes Mellitus: A qualitative study among app users and non-app users. Diabetol Metab Syndr., 11(84), 1-17.

Julian, G. S. et al. (2020). Cost of macrovascular complications in people with diabetes from a public healthcare perspective: a retrospective database study in Brazil. Journal of Medical Economics, 23(9), 985-993.

Kao, C. \& Liebovitz, D. M. (2017). Consumer Mobile Health Apps: Current State, Barriers, and Future Directions. Clinical Informatics in Physiatry, 9(5), 10615 .

Kumar, S. et al. (2018). A Diabetes Mobile App With In-App Coaching From a Certified Diabetes Educator Reduces A1C for Individuals With Type 2 Diabetes. Diabetes Educ., 44(3), 226-36.

Lechner, J., O'leary, O. E., \& Stitt, A. W. (2017). The pathology associated with diabetic retinopathy. Vision Research, 139, 7-14.

Leasher, J. L. et al. (2016). Global Estimates on the Number of People Blind or Visually Impaired by Diabetic Retinopathy: A Meta-analysis From 1990 to 2010. Diabetes Care, 39(9), 1643-9.

Polit, D. F. \& Beck, C. T. (2018). Fundamentos de pesquisa em enfermagem: avaliação de evidências para a prática da enfermagem (9a ed.). Porto Alegre: Artmed.

Razalli, N. M. \& Yap, B. (2011). Power Comparisons of Shapiro-Wilk, Kolmogorov-Smirnov, Lilliefors and Anderson-Darling Tests. J. Stat. Model. Analytics, 2(1), 21-33.

Rezende, K. F. et al. (2015). Detecção de pé em risco de ulceração usando uma nova versão do software sisped em pacientes com diabetes tipo 2 em um ambiente de atenção primária. Diabetol Metab Syndr, 7, A256. 10.1186/1758-5996-7-S1-A256

Sabanayagam, C. et al. (2019). Incidence and progression of diabetic retinopathy: a systematic review. The Lancet Diabetes and Endocrinology, 7(2), 140-9.

Sim, I. (2019). Mobile Devices and Health. N Engl J Med, 381, 956-68.

Sociedade Brasileira de Diabetes. (2015). Retinopatia diabética. https://www.diabetes.org.br/profissionais/images/pdf/diabetes-tipo-2/016-Diretrizes-SBDRetinopatia-pg149.pdf

Whitehead, L. \& Seaton, P. (2016). The Effectiveness of Self-Management Mobile Phone and Tablet Apps in Long-term Condition Management: A Systematic Review. J Med Internet Res, 18(5), e97. 10.2196/jmir.4883.

Yu, Y. et al. (2019). Effects of mobile phone application combined with or without self-monitoring of blood glucose on glycemic control in patients with diabetes: A randomized controlled trial. J Diabetes Investig., 10(5):1365-71. 